\title{
Coexistence of stable dark- and bright-soliton Kerr combs in normal-dispersion resonators
}

\author{
P. Parra-Rivas, ${ }^{1,2}$ D. Gomila, ${ }^{2}$ and L. Gelens ${ }^{1,3}$ \\ ${ }^{1}$ Applied Physics Research Group, APHY, Vrije Universiteit Brussel, 1050 Brussels, Belgium \\ ${ }^{2}$ Instituto de Física Interdisciplinar y Sistemas Complejos, IFISC (CSIC-UIB), Campus Universitat de les Illes Balears, \\ E-07122 Palma de Mallorca, Spain \\ ${ }^{3}$ Laboratory of Dynamics in Biological Systems, KU Leuven Department of Cellular and Molecular Medicine, \\ University of Leuven, B-3000 Leuven, Belgium \\ (Received 18 January 2017; published 26 May 2017)
}

\begin{abstract}
Using the Lugiato-Lefever model, we analyze the effects of third-order chromatic dispersion on the existence and stability of dark- and bright-soliton Kerr frequency combs in the normal dispersion regime. While in the absence of third-order dispersion only dark solitons exist over an extended parameter range, we find that third-order dispersion allows for stable dark and bright solitons to coexist. Reversibility is broken and the shape of the switching waves connecting the top and bottom homogeneous solutions is modified. Bright solitons come into existence thanks to the generation of oscillations in the switching-wave profiles. Temporal oscillatory instabilities of dark solitons are suppressed in the presence of sufficiently strong third-order dispersion, while bright solitons are never found to oscillate in time. As a result of third-order dispersion both bright and dark solitons are found to move with a velocity that depends on their width.
\end{abstract}

DOI: 10.1103/PhysRevA.95.053863

\section{INTRODUCTION}

The Lugiato-Lefever [1] equation (LLE) has attracted a lot of interest in the last few years for describing the generation of Kerr frequency combs in high- $Q$ microresonators driven by a continuous-wave (CW) laser [2,3]. These frequency combs can be integrated on chips [4] and used to measure time intervals and light frequencies with a exquisite accuracy, leading to numerous key applications [5-9]. In this framework a Kerr frequency comb corresponds to the frequency spectrum of a temporal dissipative structure, such as patterns or solitons, circulating inside the cavity $[10,11]$. While most theoretical studies have focused on the anomalous second-order group velocity dispersion (GVD) regime [12-14], where the typical dissipative states are bright solitons, the normal GVD regime has recently attracted interest due to the difficulty of obtaining anomalous GVD in some spectral ranges. In contrast to the anomalous regime, dark solitons are found in the normal GVD regime, i.e., low-intensity dips embedded in a highintensity homogeneous background. The bifurcation structure and temporal dynamics of these dark solitons, also called "platicons", have been recently studied [15-17], and their origin is intimately related to the locking of switching waves (SWs) connecting coexisting homogeneous state solutions of high and low intensity [16-18]. Such dissipative localized structures have also been studied in spatial systems, both in one and two dimensions, with different nonlinearities [19,20]. The generation of dark pulse Kerr frequency combs has been achieved experimentally by several groups [21-23].

In the anomalous GVD regime it was shown that highorder chromatic dispersion effects can modify the dynamics and bifurcation structure of solitons in the LLE [24,25]. In particular, third-order dispersion (TOD) generates the emission of dispersive waves that can lead to the suppression of dynamical regimes such as oscillations and chaos [26,27]. As TOD also breaks reversibility, solitons move with a constant velocity [26-30]. While recent work has numerically shown that TOD induces similar dispersive waves in dark solitons in both normal and anomalous dispersion regions [31], a complete understanding of the influence of TOD on the dynamics and bifurcation structure of dark pulse Kerr frequency combs is still lacking. The influence of TOD on single-pass, conservative systems that can be described by a generalized nonlinear Schrödinger equation has been studied before [32-34]. However, optical cavities are dissipative by nature such that the temporal structures arising here are fundamentally different to those appearing in conservative systems, and results cannot be gleaned from conservative systems in a straightforward way. Therefore, in this paper, we present a detailed analysis of the bifurcation structure of soliton Kerr combs (Kerr combs corresponding to a soliton circulating in the cavity) in the normal dispersion regime in the presence of TOD. In particular, we show that stable bright and dark solitons coexist over an increasingly wide parameter range for increasing values of TOD, and we explain such soliton stabilization by analyzing the shape of SWs whose locking lies at the heart of this phenomenon. Moreover, we show that bright and dark solitons are stable in the presence of sufficiently strong TOD, and that solitons move with a velocity that depends on their width.

\section{SOLITONS IN THE LUGIATO-LEFEVER MODEL WITH THIRD-ORDER DISPERSION}

Using the normalization of [12,35], the LLE reads

$$
\partial_{t} A=-(1+i \theta) A+i A|A|^{2}+\rho-i \partial_{\tau}^{2} A+d_{3} \partial_{\tau}^{3} A,
$$

where $t$ is the slow time describing the evolution of the intracavity field $A(t, \tau)$ on the time scale of the cavity photon lifetime, and $\tau$ is the fast time that describes the temporal structure for the field on the time scale of the resonator round trip $L$. The first term on the right-hand side describes cavity losses (the system is dissipative by nature); $\rho$ is the amplitude of the homogeneous ( $\mathrm{CW}$ ) driving field or pump; $\theta$ measures the the cavity frequency detuning between the frequency 

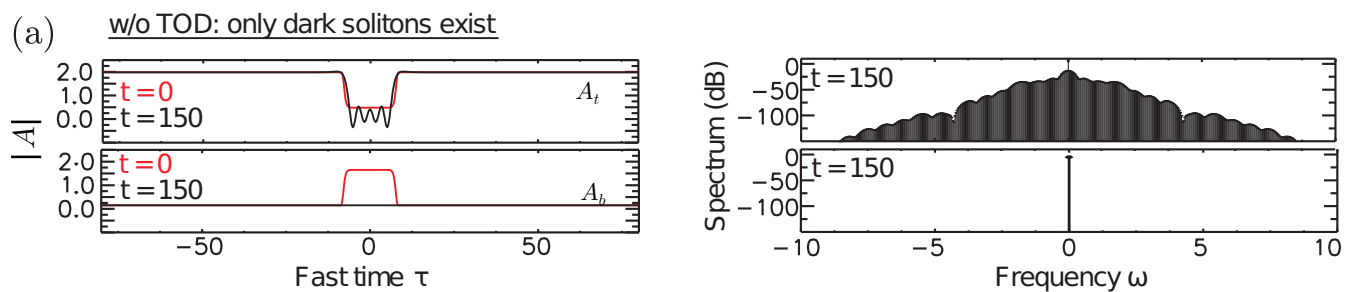

(b) w/ TOD: dark and bright solitons coexist
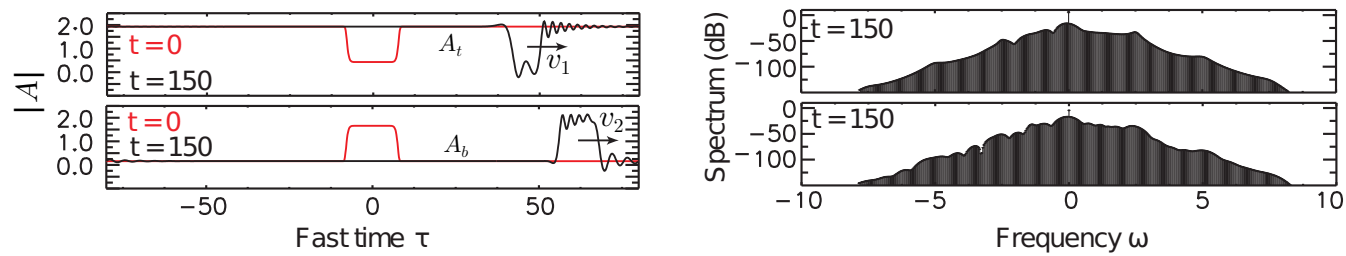

FIG. 1. Solution profile at $t=150$ (black) after time evolution in the LLE (1) of an initial dip in the top HSS $A_{t}$ or a bump on the bottom HSS $A_{b}$ (red) in the (a) absence or (b) presence of TOD. Left panels show the time profile, while the right panels show its associated comb spectrum. Parameter set: (a) $\left(\theta, \rho, d_{3}\right)=(4,2.175,0)$; (b) $\left(\theta, \rho, d_{3}\right)=(4,2.3,0.7)$.

of the input pump and the nearest cavity resonance; $\partial_{\tau}^{2}$ models the GVD (here assumed to be normal at the pump frequency); the sign of the cubic term is set so that it corresponds to the self-focusing Kerr nonlinearity; and $d_{3} \partial_{\tau}^{3}$ models the TOD and $d_{3}$ is its strength. The parameter $d_{3}$ can be determined from the physical parameters of the system using the relation

$$
d_{3} \equiv \frac{\beta_{3}}{3\left|\beta_{2}\right|} \sqrt{\frac{2 \alpha}{\left|\beta_{2}\right| l}}
$$

where $\alpha=\pi / \mathcal{F}$ is half the percentage of power lost per round trip, $\mathcal{F}$ is the cavity finesse, $l$ is the length of the cavity, and $\beta_{2}\left(\beta_{3}\right)$ is the second (third)-order dispersion coefficient. Typical values of these parameters have been listed in [27] for three different physical systems, namely, crystalline magnesium fluoride $\left(\mathrm{MgF}_{2}\right)$ and silicon nitride $\left(\mathrm{Si}_{3} \mathrm{~N}_{4}\right)$ microresonators, as well as cavities consisting of standard and dispersion-shifted optical fibers. While fiber resonators are much longer than microresonators $\left(l \approx 10^{2}\right.$ $\mathrm{m}$ vs $l \approx 10^{-4}-10^{-2} \mathrm{~m}$ ), decreasing $d_{3}$, their cavity losses are generally also much higher $\left(\alpha \approx 10^{-1}\right.$ vs $\alpha \approx 10^{-5}-10^{-2}$ ), leading to an increase of $d_{3}$. This shows that decreasing the strength of second-order dispersion $\beta_{2}$ is critical to increasing the effective strength of $\operatorname{TOD}\left(d_{3}\right)$. Using the normalization (2), it was shown that the absolute value of $d_{3}$ can realistically range anywhere from 0 to approximately 2 . Here, we will consider values of $d_{3}$ up to 0.7 .

The homogeneous steady state (HSS) solutions $A_{0}$ are given by the classic cubic equation of dispersive optical bistability, namely,

$$
I_{s}^{3}-2 \theta I_{s}^{2}+\left(1+\theta^{2}\right) I_{s}=\rho^{2},
$$

where $I_{s} \equiv\left|A_{0}\right|^{2}$. For $\theta<\sqrt{3}$, Eq. (3) is monovaluate and hence the system is monostable. However, for $\theta>\sqrt{3}$, three HSS solutions exist: $A_{t}$ (a stable HSS of higher intensity), $A_{m}$ (an unstable saddle HSS of intermediate intensity), and $A_{b}$ (a stable HSS of lower intensity). The different branches meet at saddle-nodes $\mathrm{SN}_{\text {hom,1 }}$ and $\mathrm{SN}_{\text {hom,2 }}$ located at

$$
I_{b, t}=\frac{2 \theta}{3} \pm \frac{1}{3} \sqrt{\theta^{2}-3} .
$$

Figure 1 illustrates how the system can relax into different stable structures depending on the initial condition. In the absence of TOD, Figure 1(a) shows that a dip in the highintensity HSS $A_{t}$ (red) can evolve into a stable dark soliton (black), while a bump on the low-intensity HSS $A_{b}$ (red) relaxes back to $A_{b}$. This observation that dark solitons exist, but bright solitons do not, is general [15-17]. Reference [16] discussed that dark solitons exist due to the locking of overlapping oscillatory tails in the profile of SWs connecting the upper state $A_{t}$ to the bottom state $A_{b}$. As such oscillations are absent in SWs approaching the upper state $A_{t}$, bright solitons do not exist (apart from at one single value of the pump, called the Maxwell point $\rho^{M}$ [16]). Figure 1(b) shows a similar numerical simulation, but now in the presence of TOD $\left(d_{3}=0.7\right)$. The initial condition corresponding to a dip still evolves to a dark soliton, which now has an asymmetric profile that moves at velocity $v_{1}$ while stably maintaining its temporal shape and corresponding frequency spectrum. However, in contrast to the case without TOD, an initial bump now no longer relaxes to the HSS $A_{b}$, but it forms a bright soliton corresponding to a fixed profile moving at velocity $v_{2}$.

\section{ADDITIONAL OSCILLATORY TAILS LEAD TO BRIGHT SOLITONS}

As already suggested, analyzing the shape of the SWs connecting HSSs $A_{t}$ and $A_{b}$ is the key to understanding why dark and bright solitons can stably coexist in the presence of TOD. Any steadily drifting dark or bright soliton is a solution of the time-independent LLE

$$
-(1+i \theta) A+i A|A|^{2}+\rho+v \partial_{\tau^{\prime}} A-i \partial_{\tau^{\prime}}^{2} A+d_{3} \partial_{\tau^{\prime}}^{3} A=0,
$$

where $\tau^{\prime} \equiv \tau-v t$ and the velocity $v$ is computed as part of the solutions of Eq. (5). Defining a six-dimensional phase 

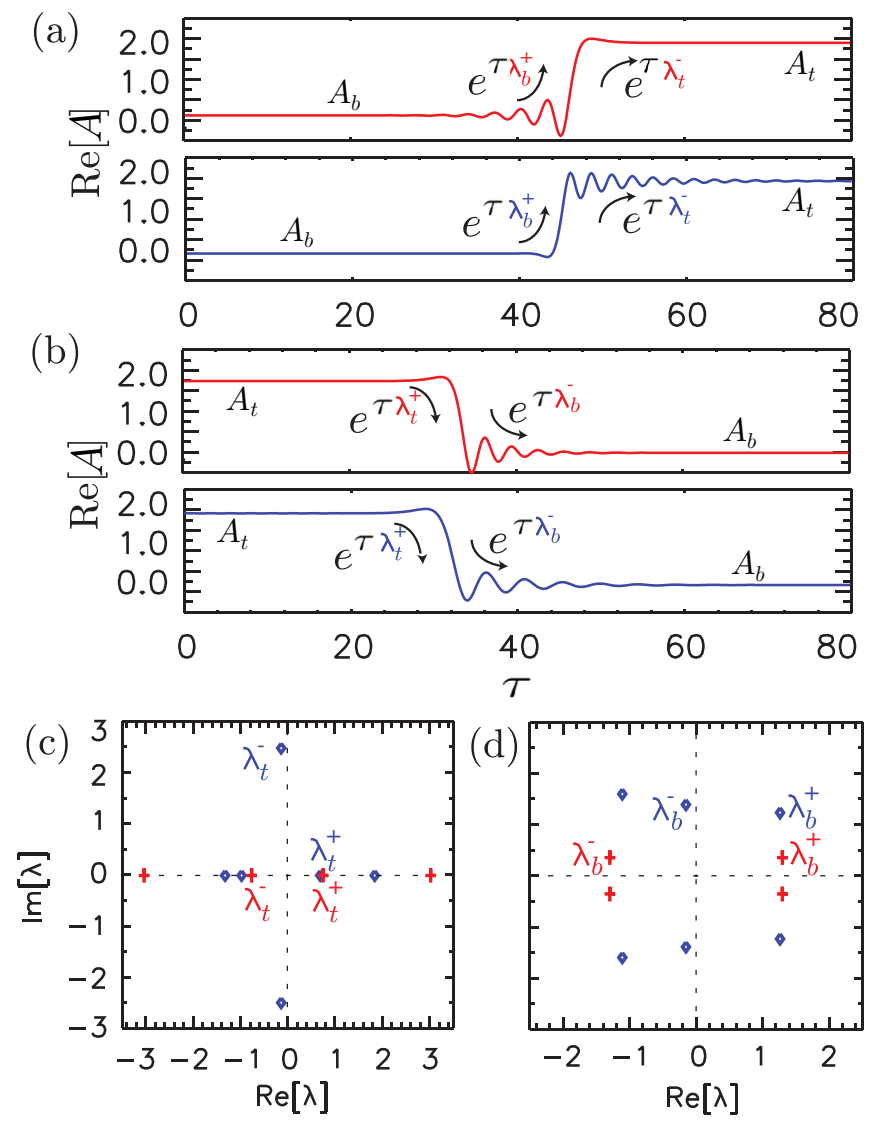

FIG. 2. Switching waves (a) $S_{u}$ and (b) $S_{d}$, in each case for $d_{3}=0$ (top panel, red) and $d_{3}=0.7$ (bottom panel, blue). The spatial eigenvalues corresponding to $A_{t}$ and $A_{b}$ are shown in (c) and (d), respectively, both for $d_{3}=0$ (red crosses) and $d_{3}=0.7$ (blue diamonds). $(\theta, \rho)=(4,2.325)$.

space by variables $u_{1}=\operatorname{Re}[A], u_{2}=\operatorname{Im}[A], u_{3}=d_{\tau^{\prime}} \operatorname{Re}[A]$, $u_{4}=d_{\tau^{\prime}} \operatorname{Im}[A], u_{5}=d_{\tau^{\prime}}^{2} \operatorname{Re}[A]$ and $u_{6}=d_{\tau^{\prime}}^{2} \operatorname{Im}[A]$, Eq. (5) can be recast to the following dynamical system:

$$
\begin{aligned}
& d_{\tau} u_{1}=u_{3} . \\
& d_{\tau} u_{2}=u_{4} . \\
& d_{\tau} u_{3}=u_{5} . \\
& d_{\tau} u_{4}=u_{6} . \\
& d_{\tau} u_{5}=d_{3}^{-1}\left[-u_{4}-v u_{3}+u_{1}-\theta u_{2}-u_{2} I_{s}-\rho\right], \\
& d_{\tau} u_{6}=d_{3}^{-1}\left[u_{3}-v u_{4}+u_{2}-\theta u_{2}-u_{1} I_{s}\right],
\end{aligned}
$$

with $I_{s}=u_{1}^{2}+u_{2}^{2}$, and where we have omitted the prime $\left(^{\prime}\right)$. In this framework any SW connecting $A_{b}$ and $A_{t}$ or vice versa corresponds to a heteroclinic trajectory connecting these HSSs in the phase space, and the shape of the SWs is determined by the linearization of Eq. (6) around both $A_{b}$ and $A_{t}$, and therefore by the spatial eigenvalues satisfying

$$
d_{3}^{2} \lambda^{6}+a_{4} \lambda^{4}-2 d_{3} \lambda^{3}+a_{2} \lambda^{2}-2 v \lambda+a_{0}=0,
$$

where $a_{4}=2 v d_{3}+1, \quad a_{2}=-4 I_{s}+2 \theta+v^{2}, \quad$ and $a_{0}=$ $4 I_{s} \theta-3 I_{s}^{2}-\theta^{2}+1[36,37]$. Alternatively, one can perform a linear stability analysis directly on Eq. (5) as shown in the Appendix. Figure 2(a) shows the shape of an up-switching wave $S_{u}$ connecting the low-intensity HSS $A_{b}$ to the highintensity HSS $A_{t}$, both in the absence (red) and presence (blue) of TOD. Figure 2(b) similarly shows the profile of the opposite down-switching wave $S_{d}$, connecting the high-intensity HSS $A_{t}$ to the low-intensity HSS $A_{b}$.

Figures 2(c) and 2(d) plot the corresponding spatial eigenvalues that are solutions of the characteristic polynomial equation (7). Close to the top HSS $A_{t}$, the eigenvalues in Fig. 2(c) show how the switching waves approach $\left(S_{u}\right)$ and leave $\left(S_{d}\right)$ the HSS $A_{t}$, both with (blue) and without (red) TOD. Such approach (departure) to (from) the top HSS $A_{t}$ can be approximated linearly as follows

$$
S^{u, d}(\tau)-A_{t} \propto e^{\lambda_{t}^{-,+} \tau},
$$

where $\lambda_{t}^{-}\left(\lambda_{t}^{+}\right)$is the spatial eigenvalue with the smallest absolute value of the real part, which is the one determining how this approach (departure) occurs asymptotically. Similarly, close to the bottom HSS $A_{b}$, the approach to and departure from $A_{b}$ is approximated by

$$
S^{u, d}(\tau)-A_{b} \propto e^{\lambda_{b}^{-,+} \tau}
$$

Without TOD, the system is reversible under the transformation $\tau \rightarrow-\tau$. As a consequence spatial eigenvalues (red) come in pairs and are symmetric with respect to both axes $\operatorname{Re}[\lambda]=0$ and $\operatorname{Im}[\lambda]=0$. This means that both $S_{u}$ [Fig. 2(a)] and $S_{d}$ [Fig. 2(b)] approach and leave $A_{t}$ and $A_{b}$ in the same way. The top HSS $A_{t}$ is always approached or left in a monotonic way, explained by the corresponding purely real spatial eigenvalues $\lambda_{t}$. In contrast, the bottom HSS $A_{b}$ is always approached or left in an oscillatory fashion, because its corresponding spatial eigenvalues $\left(\lambda_{b}\right)$ are complex. The damping rate $q$ and the frequency $\Omega$ of these oscillatory tails around $A_{b}$ correspond to the real and imaginary parts of the spatial eigenvalues $\lambda_{b}$. Such oscillations can interlock around $A_{b}$ to form stable dark solitons, but the absence of similar oscillations around $A_{t}$ prevent stable bright solitons to form.

When adding TOD, the dynamics around the HSSs are described by six eigenvalues instead of four, and they are no longer symmetric. The shapes of the SWs change in such a way that they now approach the HSS $A_{t}$ in an oscillatory way (complex $\lambda_{t}^{-}$). Therefore locking can occur not only in the bottom HSS $A_{b}$, but also in the top $A_{t}$, forming bright solitons.

\section{MODIFICATION OF THE BIFURCATION STRUCTURE OF THE SOLITONS}

The steadily drifting soliton solutions of Eq. (5) can be found numerically, and with arbitrary precision, using a Newton-Raphson method. Next, continuation techniques can be used to track those solutions as parameters are varied [38,39]. In this way it is possible to build up bifurcation diagrams, which help to understand the bifurcation structure and the organization of the different solutions that exist in the system.

However, in order to solve Eq. (5), one also needs to calculate the velocity $v$ as part of the solution, which can be done by adding an extra condition in the form of a constraint $Q[A]=0$. Here, we choose the maximum of the structure to be at a fixed position $\tau_{0}$, which can be described by the equation

$$
Q[A] \equiv \partial_{\tau}\left(|A|^{2}\right)_{\tau_{0}}=0
$$


Alternatively, one can also use integral phase conditions as done in [40]. Therefore, for any set of parameters, the solution of the system formed by both Eqs. (5) and (10) gives both the steadily drifting soliton and its velocity. In this work, we have discretized the domain using a grid of $N=1024$ points, such that Eq. (5) then becomes a set of coupled algebraic equations.

The stability of all steady-state solutions is indicated by solid (stable) or dashed (unstable) lines. We determined the stability by calculating the eigenspectrum of the linear operator $\mathcal{L}[A(\tau)]$ associated with Eq. (5), i.e., solving the eigenvalue problem

$$
\mathcal{L}\left[A_{s}(\tau)\right] \psi=\sigma \psi,
$$

with $\psi$ the eigenmode associated with the eigenvalue $\sigma$, both depending on the parameters of the system. If $\operatorname{Re}[\sigma]<0(>0)$, the states are stable (unstable), and if for a given set of parameters $\operatorname{Re}[\sigma]=0$ the system undergoes a bifurcation. Equation (11) has to be solved numerically. Hence, the linear operator corresponds to the Jacobian matrix associated with the coupled algebraic equations that originate from discretizing Eq. (5).

In blue, the three HSS solutions are shown: the stable top $\left(\mathrm{A}_{t}\right)$ and bottom $\left(\mathrm{A}_{b}\right)$ HSS, and the unstable middle HSS $\left(\mathrm{A}_{m}\right)$. The three HSS solutions are connected by two saddle-node bifurcations. In the neighborhood of the saddlenode bifurcation of the upper HSS (indicated by $\mathrm{SN}_{\mathrm{hom}, 2}$ ), an analytical asymptotic soliton solution of Eq. (5) is known for $d_{3}=0$. It has the form $A(\tau)-A_{t}=C \operatorname{sech}^{2}(B \tau)$, with $C$ and $B$ depending on the control parameters $\theta$ and $\rho$ [17]. We used this solution as an initial guess in the continuation algorithm to track these structures to any value of the parameters $\theta$ and $\rho$ (and later also $d_{3}$ ) far from $\mathrm{SN}_{\mathrm{hom}, 2}$.

The resulting bifurcation diagram is shown in Fig. 3, where we plot the mean energy

$$
\|A\|^{2} \equiv L^{-1} \int_{-L / 2}^{L / 2}|A|^{2} d \tau
$$

of HSSs and solitons as a function of the pump amplitude $\rho$ for $d_{3}=0$ and increasing values of $d_{3}$. In black, the bifurcation diagram of dark solitons is shown for $d_{3}=0$ and has been discussed in detail in $[16,17]$. Unstable dark solitons originate from the saddle-node point $\mathrm{SN}_{\mathrm{hom}, 2}$ and acquire stability at the next turning point when increasing the pump amplitude. Dark solitons of increasing width, corresponding to branches with lower mean energy, exist over a parameter range $\rho$ that becomes narrower and narrower, eventually collapsing to the Maxwell point of the system at $\rho^{M}$. These dark solitons are connected by unstable solution branches that serve to add additional spatial oscillations in their profiles, leading to the broadening of the dark states. This type of bifurcation structure is called collapsed snaking [16,17,41,42], which is significantly different from the homoclinic snaking appearing for dissipative solitons associated with subcritical patterns. Such homoclinic snaking, where many solutions coexist over a fixed parameter range around the Maxwell point, is probably better known and has been widely studied in physics [43,44] and optics [13,45-47].

When TOD is taken into account, $S_{u}$ gradually develops oscillations when increasing $d_{3}$, which allows bright solitons

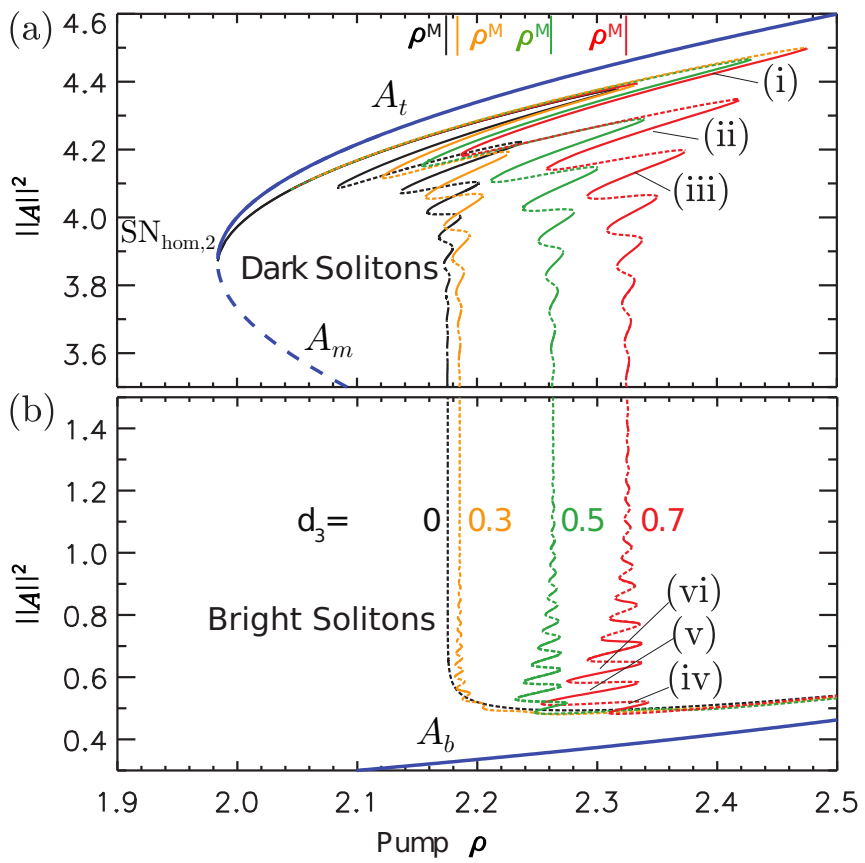

FIG. 3. Bifurcation diagrams of HSSs (blue) and dark and bright solitons for $\theta=4$ and increasing values of $d_{3}=0$ (black), 0.3 (yellow), 0.5 (green), 0.7 (red). Solid (dashed) lines correspond to stable (unstable) states.

to come into existence. This can be seen in Fig. 3(b) in yellow $\left(d_{3}=0.3\right)$, and bright solitons now exist over a narrow range of pump values $\rho$. Increasing $d_{3}$ further, the spatial oscillations in $S_{u}$ become stronger, leading to the existence of bright solitons over a wider range of pump parameters $\left[d_{3}=0.5\right.$ (green), 0.7 (red)]. Figure 3(a) shows that also the dark solitons exist over a wider range of parameters, and the Maxwell point $\rho^{M}$ (around which the collapsed snaking structure is organized) shifts to higher values of the pump $\rho$. Typical solution profiles of solitons corresponding to different branches are plotted in Fig. 4 for $d_{3}=0.7$ (red). In the presence of TOD, both dark [Figs. 4(i)-4(iii)] and bright [Figs. 4(iv)-4(vi)] solitons exist over an increasingly narrow parameter range as they increase their width. As the solitons become wider by incorporating additional oscillations, their corresponding frequency comb spectrum also becomes more complex and modulated. This complexity in the comb spectra is associated with the oscillatory profile close to each HSS $\left(A_{t}\right.$ and $\left.A_{b}\right)$. The comb width, however, is not largely affected because it is mainly controlled by the sharpest and most pronounced feature in the soliton profile, being the fast switching between both HSSs, which remains approximately the same for all soliton solutions.

\section{SUPPRESSION OF TEMPORAL SOLITON INSTABILITIES}

For $d_{3}=0$, at higher values of the detuning $\theta$, dark solitons have been shown to undergo Hopf instabilities and period doubling bifurcations starting a route to temporal chaos $[16,17]$. This scenario is similar to the one regarding bright solitons in the anomalous dispersion regime $[12,13]$. In 

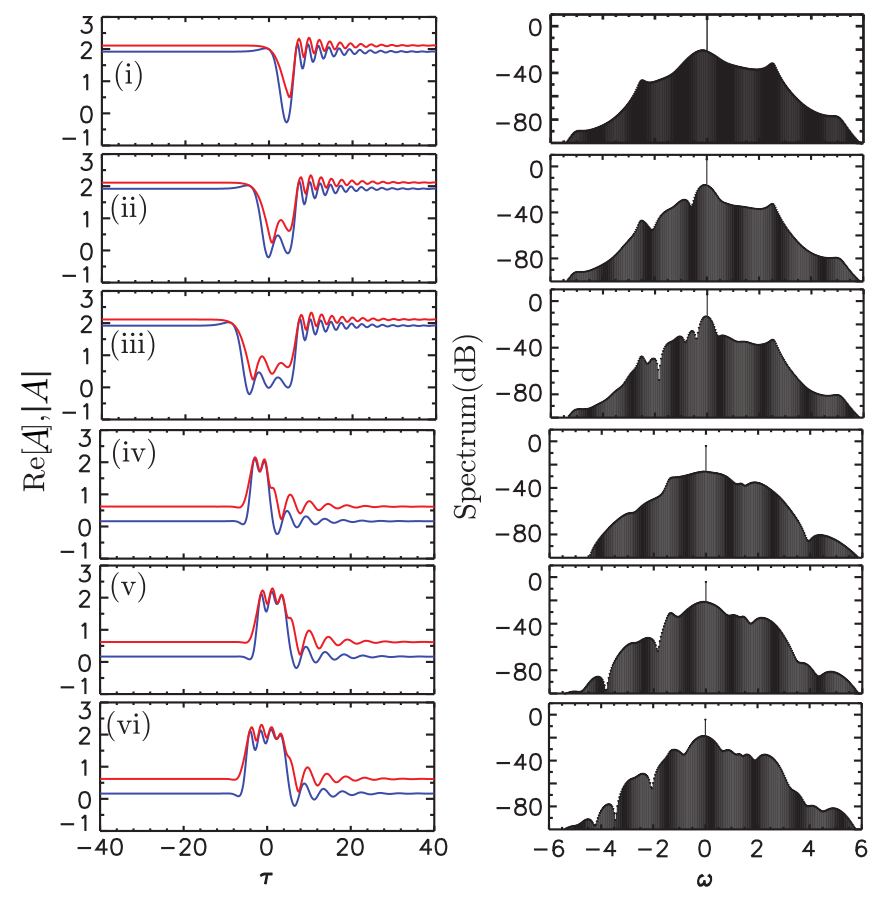

FIG. 4. Temporal profiles [left; $\operatorname{Re}(A)$ in blue, $|A|$ in red] and spectral intensities (right, in $\mathrm{dB}$ ) of asymmetric dark and bright solitons corresponding to the locations (i)-(iii) and (iv)-(vi) in Fig. 3. $\left(\theta, \rho, d_{3}\right)=(4,2.232,0.7)$.

the anomalous dispersion regime, such temporal oscillations, also called "breathers", were experimentally first observed in fiber resonators [12] and have recently also been measured in microresonators [48-50]. In the anomalous regime, it was furthermore shown that TOD, which leads to drift instabilities, could suppress such oscillatory and chaotic temporal dynamics of bright solitons [26,27].

We found that this mechanism of stabilization is also present in the normal dispersion regime. We characterized the Hopf bifurcations leading to temporal oscillations by calculating numerically when the eigenspectrum has a pair of purely imaginary complex conjugate eigenvalues $\sigma= \pm i \omega$. At such Hopf bifurcation, solitons start to oscillate in amplitude with frequency $\omega$. We then tracked these Hopf bifurcations in parameter space to find the oscillatory regions and to see how they change when modifying $d_{3}$.

To illustrate this we first show the bifurcation diagram for $\theta=5$ and $d_{3}=0$ in Fig. 5(a). Narrow dark solitons are unstable between the Hopf bifurcations $\mathrm{H}_{1}$ and $\mathrm{H}_{2}$ leading to temporal oscillations. When $d_{3} \neq 0$, the Hopf bifurcations shift in such way that the oscillatory region shrinks for increasing values of TOD until it disappears [see Figs. 5(b)-5(d) for $d_{3}=0.2,0.3$ and 0.7, respectively). While for $d_{3}=0.2$, dark solitons between $\mathrm{H}_{1}$ and $\mathrm{H}_{2}$ oscillate and drift, for $d_{3}=0.3$ and 0.7 the oscillatory instabilities have been suppressed and only a drifting soliton remains. The direction in which the soliton drifts is not obvious by looking at its profile and changes with the pump $\rho$. In Fig. 5, solid purple (green) lines correspond to dark solitons with positive (negative) velocity, while the solid red lines (for $d_{3}=0$ ) indicate zero velocity. By increasing the strength of TOD, the parameter range of solitons with

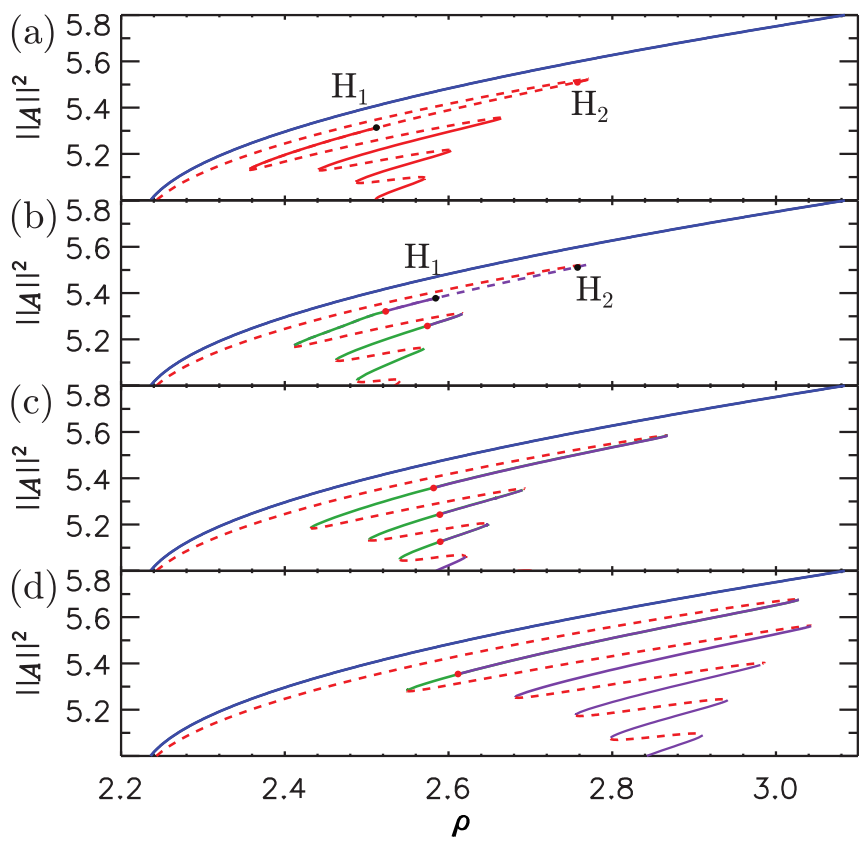

FIG. 5. Bifurcation diagrams for $\theta=5$ at different values of $d_{3}=0$ (a), 0.2 (b), 0.3 (c), 0.7 (d). $\mathrm{H}_{1,2}$ correspond to Hopf bifurcations (black dots). Solid (dashed) lines stand for stable (unstable) states. In solid purple (green) lines we refer to states with positive (negative) velocity. Solid red lines (for $d_{3}=0$ ) and red dots indicate zero velocity.

negative velocity shrinks. Although bright solitons could in principle also undergo oscillatory instabilities and experience similar stabilization with increasing TOD, for the parameter range considered in this work, no such oscillations of bright solitons have been found. Despite the absence of oscillatory instabilities, both bright and dark solitons drift with positive or negative velocity whenever $d_{3} \neq 0$, which we explore in more detail in the next section. Whereas temporal oscillations in the soliton profile also lead to an oscillating frequency comb spectrum, a steady soliton drift does not affect the comb profile, which remains stable in time.

\section{SOLITON VELOCITY}

When $d_{3} \neq 0$, the system is no longer $\tau$ reversible due to the introduction of an odd derivative in the right-hand side of Eq. (1). Due to this broken reversibility, the solutions of Eq. (1) are asymmetric with respect to the center of the structure, let us say $\tau=0$, and they drift with a constant velocity $v$. The breaking of the reflection symmetry causes a modification of the solution profile related to the shape of the Goldstone mode, which induces a permanent shift of the solution (i.e., a drift), and an asymmetry of such a state [51]. In the linear regime it is possible to show, using perturbation theory, that $v \propto d_{3}$, although this result is only valid for small values of TOD, i.e., $\left(d_{3} \ll 1\right)[40]$.

However, as mentioned in Sec. IV, the velocity can also be calculated via the Newton-Raphson method as part of the solution of the system formed by Eqs. (5) and (10). This velocity is shown for $\theta=4$ (no oscillatory instabilities) in 


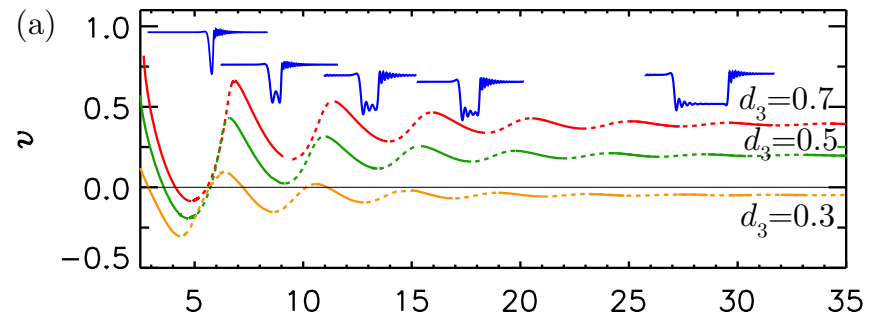

(b)

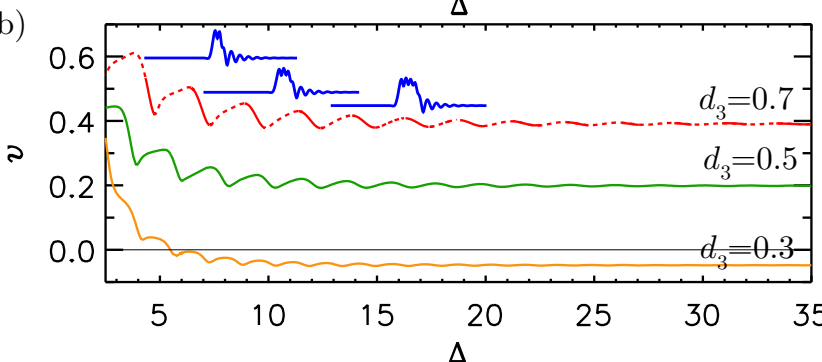

FIG. 6. (a) Velocity of dark solitons as a function of the width at half minimum $\Delta$ for $\theta=4$ and $d_{3}=0.3,0,5,0.7$. The velocity changes in a damped oscillatory way as a function of $\Delta$. The stable (unstable) dark solitons correspond to the solid (dashed) lines. (b) Velocity of the bright solitons for the same parameter values.

Fig. 6 for both dark [Fig. 6(a)] and bright [Fig. 6(b)] solitons as a function of its width at half minimum and/or maximum $\Delta$. For dark solitons Fig. 6(a)], one can see how the velocity of the dark solitons oscillates in $\Delta$ for three different values of TOD $\left(d_{3}=0.3,0.5,0.7\right)$. In all three cases, the difference between the minimum and maximum velocity of a soliton decreases as it gets wider, approaching a constant value for very wide dark solitons (large $\Delta$ ). This constant velocity in the limit of very wide structures increases with the TOD. In the case of bright solitons [Fig. 6(b)], the velocity oscillates similarly with $\Delta$. In this case, however, the oscillation period in $\Delta$ is smaller, as the spatial oscillations are faster than for the dark solitons. This difference in spatial oscillations can be clearly seen in the solution profiles in Fig. 4. The calculated velocity shown in Fig. 6 is to be interpreted as a drift in the fast time scale of the resonator round trip (where $\tau$ is scaled by $\sqrt{2 \alpha} / \sqrt{\left|\beta_{2}\right| l}$ ) per time unit $t$ (where $t$ depends on the photon lifetime as $t \times \alpha / t_{r}$ with $t_{r}$ the round-trip time) [12,35]. This drift velocity thus strongly depends on $\alpha$ and $l$, which can change significantly from device to device (see, e.g., [27]).

As solitons of different widths travel at different speeds, they eventually collide in a periodic domain and are forced to interact with one another. This is illustrated in Fig. 7 for two dark solitons traveling in opposite directions. As these solitons are dissipative structures, they do not come out of such a collision unchanged such as the classical solitons in conservative systems. A number of outcomes are possible. In Fig. 7(a) we show the formation of a bound state, while in Fig. 7(b) we illustrate how they merge to form a new wider soliton. Note that even if the solitons travel in the same direction, but with different velocity, they will interact in a similar way. For bright solitons, similar types of behavior are found. It is a priori not obvious to know which type of structure will emerge from the collision. A detailed characterization of such interactions is beyond the scope of this work. For a more
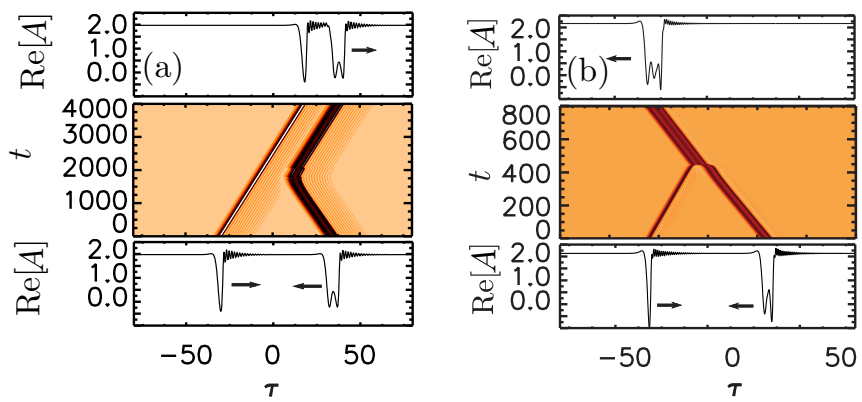

FIG. 7. Time evolution of two dark solitons traveling at different speeds for (a) $\left(\theta=4, d_{3}=0.3, \rho=2.55\right)$ and (b) $(\theta=5$, $d_{3}=0.2, \rho=2.55$ ).

in-depth theoretical and experimental study of bound states of solitons in the presence of TOD in the Lugiato-Lefever equation, we refer to $[52,53]$. In these works, solitons interact through their oscillatory tails and bound states can form as a result of the stable locking of such tails. We note that the interaction illustrated in Fig. 7 is initially driven by the inherent velocity of each soliton, whereas the final formation of bound states is controlled by the interacting oscillatory tails and soliton cores. Such interaction of solitons over very long distances has also been shown experimentally, where the soliton movement was induced via acoustic waves rather than TOD [54].

\section{CONCLUSIONS}

In summary, we have presented a bifurcation analysis of solitons and their corresponding Kerr combs in the normal group velocity dispersion regime in the presence of third-order chromatic dispersion. Bright solitons have been shown to coexist with dark solitons over a wide parameter region due to the locking of switching waves. Third-order dispersion induces oscillatory tails in the switching waves close to the high-intensity homogeneous solution, allowing bright solitons to form over an extended range of pump powers, which was not possible without third-order dispersion. Both dark and bright solitons are organized in a collapsed snaking bifurcation diagram, such that broader solitons always exist over a more narrow parameter region. Furthermore, third-order dispersion can suppress oscillatory instabilities of dark solitons in a similar fashion as for the anomalous case. No oscillatory instabilities were found for bright solitons in the parameter range considered in this work. Finally, we have shown that dark and bright solitons of different widths propagate at different velocities, and multiple different solitons in a resonator thus eventually collide inelastically to form new solitons.

\section{ACKNOWLEDGMENTS}

We acknowledge support from the Research FoundationFlanders (FWO-Vlaanderen) (P.P.-R.), the Belgian Science Policy Office (BelSPO) under Grant No. IAP 7-35, the Research Council of the Vrije Universiteit Brussel, and Ministerio de Economía y Competitividad (Spain) and Fondo Europeo de Desarrollo Regional under Project ESoTECoS FIS2015-63628-C2-1-R (MINECO/FEDER) (D.G.). 


\section{APPENDIX}

The spatial eigenvalues can be obtained by inserting $u_{1}(\tau)=U_{s}+\epsilon U(\tau)$ and $u_{2}(\tau)=V_{s}+\epsilon V(\tau)$ into Eq. (5), where $U_{s}$ and $V_{s}$ correspond to the real and imaginary parts of the HSS solutions $A_{b}$ or $A_{t}$, and with the Ansätze $U(\tau)=a e^{\lambda \tau}, V(\tau)=b e^{\lambda \tau}$, and $\lambda \in \mathbb{C}$. Then the system of two ordinary differential equations obtained for $U(\tau)$ and $V(\tau)$ has a nontrivial solution if the next determinants satisfy

$$
\left|\begin{array}{cc}
d_{3} \lambda^{3}+v \lambda-\left(1+2 U_{s} V_{s}\right) & \lambda^{2}+\theta-3 V_{s}^{2}-U_{s}^{2} \\
-\lambda^{2}-\theta+3 U_{s}^{2}+V_{s}^{2} & d_{3} \lambda^{3}+v \lambda-\left(1-2 U_{s} V_{s}\right)
\end{array}\right|=0,
$$

which yields the same characteristic equation (7). Nonetheless we prefer using the "spatial" dynamics form (6) because it allows us to apply the theory of dynamical systems to study the formation of localized temporal structures and SWs.

[1] L. A. Lugiato and R. Lefever, Phys. Rev. Lett. 58, 2209 (1987).

[2] P. Del'Haye, A. Schliesser, O. Arcizet, T. Wilken, R. Holzwarth, and T. J. Kippenberg, Nature 450, 1214 (2007).

[3] T. J. Kippenberg, R. Holzwarth, and S. A. Diddams, Science 332, 555 (2011).

[4] V. Brasch, M. Geiselmann, T. Herr, G. Lihachev, M. H. P. Pfeiffer, M. L. Gorodetsky, and T. J. Kippenberg, Science 351, 357 (2016).

[5] Y. Okawachi, K. Saha, J. S. Levy, Y. H. Wen, M. Lipson, and A. L. Gaeta, Opt. Lett. 36, 3398 (2011).

[6] S. B. Papp, K. Beha, P. Del'Haye, F. Quinlan, H. Lee, K. J. Vahala, and S. A. Diddams, Optica 1, 10 (2014).

[7] F. Ferdous, H. Miao, D. E. Leaird, K. Srinivasan, J. Wang, L. Chen, L. T. Varghese, and A. M. Weiner, Nat. Photon. 5, 770 (2011).

[8] T. Herr, K. Hartinger, J. Riemensberger, C. Y. Wang, E. Gavartin, R. Holzwarth, M. L. Gorodetsky, and T. J. Kippenberg, Nat. Photon. 6, 480 (2012).

[9] J. Pfeifle, V. Brasch, M. Lauermann, Y. Yu, D. Wegner, T. Herr, K. Hartinger, P. Schindler, J. Li, D. Hillerkuss, R. Schmogrow, C. Weimann, R. Holzwarth, W. Freude, J. Leuthold, T. J. Kippenberg, and C. Koos, Nat. Photon. 8, 375 (2014).

[10] S. Coen, H. G. Randle, T. Sylvestre, and M. Erkintalo, Opt. Lett. 38, 37 (2013).

[11] Y. K. Chembo and C. R. Menyuk, Phys. Rev. A 87, 053852 (2013).

[12] F. Leo, L. Gelens, P. Emplit, M. Haelterman, and S. Coen, Opt. Express 21, 9180 (2013).

[13] P. Parra-Rivas, D. Gomila, M. A. Matías, S. Coen, and L. Gelens, Phys. Rev. A 89, 043813 (2014).

[14] C. Godey, I. V. Balakireva, A. Coillet, and Y. K. Chembo, Phys. Rev. A 89, 063814 (2014).

[15] V. E. Lobanov, G. Lihachev, T. J. Kippenberg, and M. L. Gorodetsky, Opt. Express 23, 7713 (2015).

[16] P. Parra-Rivas, D. Gomila, E. Knobloch, S. Coen, and L. Gelens, Opt. Lett. 41, 2402 (2016).

[17] P. Parra-Rivas, E. Knobloch, D. Gomila, and L. Gelens, Phys. Rev. A 93, 063839 (2016).

[18] S. Coen, M. Tlidi, Ph. Emplit, and M. Haelterman, Phys. Rev. Lett. 83, 2328 (1999).

[19] G. L. Oppo, A. J. Scroggie, and W. J. Firth, Phys. Rev. E 63, 066209 (2001).
[20] M. Pesch, W. Lange, D. Gomila, T. Ackemann, W. J. Firth, and G. L. Oppo, Phys. Rev. Lett. 99, 153902 (2007).

[21] W. Liang, A. A. Savchenkov, V. S. Ilchenko, D. Eliyahu, D. Seidel, A. B. Matsko, and L. Maleki, Opt. Lett. 39, 2920 (2014).

[22] S.-W. Huang, H. Zhou, J. Yang, J. F. McMillan, A. Matsko, M. Yu, D.-L. Kwong, L. Maleki, and C. W. Wong, Phys. Rev. Lett. 114, 053901 (2015).

[23] X. Xue, Y. Xuan, Y. Liu, P.-H. Wang, S. Chen, J. Wang, D. E. Leaird, M. Qi, and A. M. Weiner, Nat. Photon. 9, 594 (2015).

[24] M. Tlidi and L. Gelens, Opt. Lett. 35, 306 (2010).

[25] M. Tlidi, L. Bahloul, L. Cherbi, A. Hariz, and S. Coulibaly, Phys. Rev. A 88, 035802 (2013).

[26] C. Milián and D. Skryabin, Opt. Express 22, 3732 (2014).

[27] P. Parra-Rivas, D. Gomila, F. Leo, S. Coen, and L. Gelens, Opt. Lett. 39, 2971 (2014).

[28] A. Mussot, E. Louvergneaux, N. Akhmediev, F. Reynaud, L. Delage, and M. Taki, Phys. Rev. Lett. 101, 113904 (2008).

[29] F. Leo, A. Mussot, P. Kockaert, Ph. Emplit, M. Haelterman, and M. Taki, Phys. Rev. Lett. 110, 104103 (2013).

[30] J. K. Jang, S. G. Murdoch, S. Coen, and M. Erkintalo, Observation of Dispersive-Wave Emission by Temporal Cavity Solitons (CLEO/Europe, Munich, Germany, 2013), pp. PD-B.7.

[31] Y. He, S. Wang, and X. Zeng, IEEE, Phot. J. 8, 6 (2016).

[32] M. Conforti and Stefano Trillo, Opt. Lett. 38,3815 (2013).

[33] K. E. Webb, Y. Q. Xu, M. Erkintalo, and S. G. Murdoch, Opt. Lett. 38, 151 (2013).

[34] A. Demircan, M. Pietrzyk, and U. Bandelow, Opt. Quantum Electron. 40, 455 (2008).

[35] F. Leo, S. Coen, P. Kockaert, S.-P. Gorza, Ph. Emplit, and M. Haelterman, Nat. Photon. 4, 471 (2010).

[36] P. Colet, M. A. Matías, L. Gelens, and D. Gomila, Phys. Rev. E 89, 012914 (2014).

[37] L. Gelens, M. A. Matías, D. Gomila, T. Dorissen, and P. Colet, Phys. Rev. E 89, 012915 (2014).

[38] E. J. Doedel, Cong. Numer. 30, 265 (1981).

[39] J. M. McSloy, W. J. Firth, G. K. Harkness, and G.-L. Oppo, Phys. Rev. E 66, 046606 (2002).

[40] A. J. Scroggie, D. Gomila, W. J. Firth, and G. L. Oppo, Appl. Phys. B 81, 963 (2005).

[41] J. Knobloch and T. Wagenknecht, Physica D (Amsterdam) 206, 82 (2005). 
[42] Y.-P. Ma, J. Burke, and E. Knobloch, Physica D (Amsterdam) 239, 1867 (2010).

[43] A. R. Champneys, Physica D (Amsterdam) 112, 158 (1998).

[44] J. Burke and E. Knobloch, Phys. Rev. E 73, 056211 (2006).

[45] D. Gomila, A. J. Scroggie, and W. J. Firth, Physica D (Amsterdam) 227, 70 (2007).

[46] W. J. Firth, L. Columbo, and A. J. Scroggie, Phys. Rev. Lett. 99, 104503 (2007)

[47] S. Barbay, X. Hachair, T. Elsass, I. Sagnes, and R. Kuszelewicz, Phys. Rev. Lett. 101, 253902 (2008).

[48] C. Bao, J. A. Jaramillo-Villegas, Y. Xuan, D. E. Leaird, M. Qi, and A. M. Weiner, Phys. Rev. Lett. 117, 163901 (2016).
[49] M. Yu, J. K. Jang, Y. Okawachi, A. G. Griffith, K. Luke, S. A. Miller, X. Ji, M. Lipson, and A. L. Gaeta, Nat. Commun. 8, 14569 (2017).

[50] E. Lucas, M. Karpov, H. Guo, M. Gorodetsky, and T. Kippenberg, arXiv:1611.06567.

[51] S. V. Gurevich and R. Friedrich, Math. Model. Nat. Phenom. 8, 84 (2013).

[52] P. Parra-Rivas, D. Gomila, P. Colet, and L. Gelens, arXiv: 1705.02619.

[53] Y. Wang, F. Leo, J. Fatome, M. Erkintalo, S. G. Murdoch, and S. Coen, arXiv:1703.10604.

[54] J. K. Jang, M. Erkintalo, S. G. Murdoch, and S. Coen, Nat. Photon. 7, 657 (2013). 